\title{
Increased metastatic potential of residual carcinoma after transarterial embolization in rat with McA-RH7777 hepatoma
}

\author{
GUANG-ZHI WANG $^{1 *}$, ZHU-TING FANG $^{1,2^{*}}$, WEI ZHANG ${ }^{1}$, \\ XU-DONG QU ${ }^{1}$, SHENG QIAN ${ }^{1}$, RONG LIU ${ }^{1}$ and JIAN-HUA WANG ${ }^{1}$ \\ ${ }^{1}$ Department of Interventional Radiology, Zhongshan Hospital, Fudan University, Shanghai; ${ }^{2}$ Department of Intervention \\ Radiology, Provincial Hospital of Fujian Province, Teaching Hospital of Fujian Medical University, Fuzhou, P.R. China
}

Received September 9, 2013; Accepted October 1, 2013

DOI: $10.3892 /$ or.2013.2820

\begin{abstract}
Transarterial chemoembolization represents a firstline non-curative therapy for hepatocellular carcinoma (HCC), although the biological changes in the remaining cancer after embolization are not completely understood. In the present study, we examined whether transarterial embolization (TAE) enhances the metastatic potential of residual $\mathrm{HCC}$ and investigated the mechanisms underlying embolization. The hepatoma cell line McA-RH7777, which is marked by green fluorescent protein (GFP), was used in the study. The invasion of cells cultured under hypoxia and normoxia was observed using the Transwell assay. Twenty male buffalo rats were implanted with GFP transfected McA-RH7777 tumors in the left lateral lobe of the liver. After laparotomy and retrograde placement of a catheter into the gastroduodenal artery (on the 14th day after implantation), TAE using lipiodol $(0.2 \mathrm{ml} / \mathrm{kg}$ ) was performed. Tumor volumes were measured before and after treatment using magnetic resonance imaging (MRI). Lung metastases were observed using fluorescence imaging, and the molecular changes of residual tumor cells were evaluated by western blotting or immunohistochemistry. The invasion assays indicated that the number of invading hypoxic cells was significantly higher than that of normoxic cells $(30.2 \pm 2.46$ vs. $20.4 \pm 1.89$, $\mathrm{P}=0.013)$. Accompanying an increase in hypoxia-inducible factor-1 $\alpha$ (HIF-1 $\alpha$ ) expression, the metastatic potential of tumor cells following hypoxia or TAE was enhanced. This enhanced metastatic potential was indicated by a significant reduction in the expression of E-cadherin and an upregulation of $\mathrm{N}$-cadherin and vimentin expression. The number of lung metastases in the TAE group was $19.20 \pm 1.76$, whereas this number was $11.30 \pm 1.54$ in the control group, which
\end{abstract}

Correspondence to: Dr Rong Liu or Dr Jian-Hua Wang, Department of Interventional Radiology, Zhongshan Hospital, Fudan University, No. 180 Fenglin Road, Xuhui, Shanghai, P.R. China

E-mail: liu.rong@zs-hospital.sh.cn

E-mail:wang.jianhua@zs-hospital.sh.cn

${ }^{*}$ Contributed equally

Key words: epithelial-mesenchymal transition, embolization, hepatocellular carcinoma, hypoxia-inducible factor- $1 \alpha$, metastasis represented a statistically significant difference $(\mathrm{P}=0.003)$. In conclusion, hypoxia in the residual tumor after TAE can increase the invasiveness and metastatic potential of HCC and may be responsible for the failure of TAE.

\section{Introduction}

Hepatocellular carcinoma (HCC) is one of the most common malignancies worldwide and its treatment may be complicated by underlying cirrhosis, multiple lesions, the invasion of vital structures within the porta hepatis, or the involvement of organs outside the liver (1-3). Surgical procedures such as hepatic resection or liver transplantation represent radical treatment modalities for HCC patients. Local ablation can substitute for resection in patients at early stages for whom surgical therapies are not suitable $(4,5)$. For intermediateadvanced HCC, there are many potential therapeutic options, but their effects are limited due to the cancer's highly variable biologic behavior and morphology. Recent investigations have highlighted the utility of combination molecular targeted therapy $(6,7)$. However, the high incidence of tumor recurrence and metastasis remains a major problem that contributes to the high mortality rate, which indicates that the therapeutic strategies against HCC requires further investigation $(8,9)$.

Transarterial chemoembolization (TACE) is widely used as a palliative treatment for patients with primary or recurrent HCC and has shown encouraging results in terms of survival $(10,11)$. The rationale for transarterial embolization (TAE) is based on occluding the blood flow to the tumor and inducing tumor necrosis while preserving adequate liver function. It is generally accepted that TAE rarely achieves total necrosis of the targeted liver tumor and the behavior of more aggressive residual tumors could detract from the merit of TAE. Multiple and diverse mechanisms for this effect have been proposed, and there are considerable data indicating that elevated expression of hypoxia-inducible factor- $1 \alpha(\mathrm{HIF}-1 \alpha)$ is induced after TACE and is associated with poor prognosis in HCC patients (12). Recent studies have further demonstrated that local hypoxia induced by embolization, through activation of the HIF-1 $\alpha$ transcription factor and the resulting increased expression of vascular endothelial growth factor (VEGF), represents an important driving force in tumor progression $(13,14)$. Currently, increasing evidence suggests that TACE for 
HCC may accelerate the development of invasion and metastasis $(14,15)$. Generally, the loss of cell-to-cell contacts and the gain of motile and invasive abilities are essential prerequisites for metastasis (16). These phenotypic changes in tumor cells, designated as the epithelial to mesenchymal transition (EMT), have been described in different types of carcinoma cells including HCC $(17,18)$. EMT, which is a physiological process that also occurs during embryological development, is characterized by loss of the epithelial marker E-cadherin and increased expression of mesenchymal markers, such as $\mathrm{N}$-cadherin and vimentin. In adult organisms, EMT can be involved in tissue repair such as wound healing and the development of diseases such as chronic inflammation and carcinoma progression (19-21). In recent years, EMT has been regarded as a critical step in tumor invasion and metastasis $(22,23)$. Furthermore, accumulating evidence indicates that local hypoxia may be related to the invasiveness and metastatic potential of HCC after embolization, although the altered metastatic potential of residual cancer after TACE is not completely understood.

Therefore, we designed the present study to investigate whether TAE could enhance the metastatic potential of residual HCC and explore the relationship between EMT and TAE in vivo and in vitro.

\section{Materials and methods}

Cell culture. The Buffalo rat hepatoma cell line McA-RH7777 was obtained from the American Type Culture Collection (no. CRL1601; ATCC, Manassas, VA, USA). Transfection of McA-RH7777 cells with a lentiviral gene-transfer vector encoding the green fluorescent protein (GFP) sequence (constructed by GeneChem, Shanghai, China) resulted in dramatic GFP expression, which was confirmed by sequencing. The following experiments were performed with sorted $\mathrm{GFP}^{+}$ cells, and cells were cultivated in Dulbecco's modified Eagle's medium (DMEM; Gibco-Life Technologies, Inc., Carlsbad, CA, USA) containing $10 \%$ heat-inactivated fetal bovine serum (FBS; Gibco), $100 \mathrm{U} / \mathrm{ml}$ penicillin $\mathrm{G}$ and $100 \mathrm{mg} / \mathrm{ml}$ streptomycin at $37^{\circ} \mathrm{C}$ in a humidified incubator with $5 \% \mathrm{CO}_{2}$.

Chemically induced hypoxia and cell growth in vitro. Hypoxic conditions were achieved by exposing normoxic cells to cobalt chloride $\left(\mathrm{CoCl}_{2}, 100 \mu \mathrm{M} / \mathrm{l}\right)$, which is known to activate hypoxia-dependent pathways under normal oxygen levels by stabilizing HIF-1 $\alpha$ in a number of cell lines (24). Cells ( $2 \times 10^{3} /$ well) were seeded in triplicate in 96-well microplates and incubated under normoxic or hypoxic conditions. After 24 and $48 \mathrm{~h}$, cell proliferation was determined in triplicate using the Cell Counting kit-8 assay (CCK-8; Dojindo Laboratories, Tokyo, Japan). The results were expressed as the corrected absorbance $\left(\mathrm{A}_{570 \mathrm{~nm}}\right)$, which represented the actual absorbance of each well recorded at $570 \mathrm{~nm}$.

Cell invasion assays. Cell invasion was assessed using 24-well BioCoat Matrigel Invasion Chambers (Corning, Cambridge, UK). A total of $1 \times 10^{5}$ cells was plated onto Matrigel-coated filters, and $750 \mu \mathrm{l}$ DMEM containing 10\% FBS was added to the lower chamber of each well. After $48 \mathrm{~h}$ under hypoxic or normoxic culture conditions, cells that had reached the underside of the membrane were stained with Giemsa (Sigma). Invading cells in 3 adjacent microscope fields for each membrane were counted at x200 magnification under a microscope (Nikon, Tokyo, Japan).

Experimental animals. Buffalo rats (8-weeks old, initially weighing $201.67 \pm 3.77 \mathrm{~g}$, ranging from 181 to $222 \mathrm{~g}$ ) were obtained from Charles River Laboratories (Davis, CA, USA). All animals were maintained in the specific pathogenfree facility of the Animal Experimental Center of Fudan University and were provided with rodent chow and tap water ad libitum. Studies were conducted in accordance with the animal care policy of the Fudan University and the Animal Research Committee.

Establishment of the rat tumor model. $\mathrm{GFP}^{+} \mathrm{McA}-\mathrm{RH} 7777$ cells $\left(1 \times 10^{6}\right)$ were injected subcutaneously into the right hindlimbs of Buffalo rats. The subcutaneous tumors were removed when they reached $\sim 1 \mathrm{~cm}$ in length $(\sim 1$ month after injection) and were then minced into small cubes of $\sim 2 \times 2 \times 2 \mathrm{~mm}^{3}$. Pieces of this tumor tissue were then transplanted into the left lobe of the livers of 20 male Buffalo rats to induce HCC. Tumor implantation was performed using a modification of the technique previously described (25).

Interventional procedures. Two weeks after orthotopic implantation, a second laparotomy (following the 2nd MRI) was performed for TAE therapy. Twenty rats were randomly assigned to either the TAE group or the sham group. A silastic catheter (Dow Corning, Midland, MI, USA) with an inner diameter of $0.28 \mathrm{~mm}$ and an outer diameter $0.61 \mathrm{~mm}$ was used for catheterization. Using a binocular operative microscope (SMZ1500; Nikon), the catheter was inserted in a retrograde manner into the gastroduodenal artery and pushed forward to the opening of the common hepatic artery (Fig. 1) (26). Then embolization was performed as follow. In the TAE group, the rats received an intra-arterial injection of iodized oil $(0.2 \mathrm{ml} / \mathrm{kg})$, which had been mixed with double the volume of saline. In the sham group, rats received an equivalent amount of saline via intra-arterial injection (final volume, $0.6 \mathrm{ml} / \mathrm{kg}$ ). The entire injection process was observed under the microscope to ensure that the embolic agent was delivered into the liver. The iodized oil was washed into the proper hepatic artery via the blood flow from the common hepatic artery. The gastroduodenal artery was then fixed with a silk suture and the abdominal wall was closed. The animals were kept under standard conditions after treatment. None of the animals died during tumor cell implantation or surgery, and we observed no fistulas or injuries to the bile ducts, vessels or other surrounding structures during treatment.

Tumor growth and lung metastasis analysis. To assess tumor growth, magnetic resonance imaging (MRI) were performed by 3.0 T Magnetom (Avanto Trio, Siemens, Germany) with small animal body coil (Siemens). All animals underwent a $\mathrm{T}_{2}$-weighted imaging $\left(\mathrm{T}_{2} \mathrm{WI}\right)$ every 7 days after inoculation with a multi-slice acquisition providing complete coverage of the entire liver. $\mathrm{T}_{2} \mathrm{WI}$ is acquired using TSE BLADE technique, $70 \mathrm{~mm}$ field of view (FOV), $1.5 \mathrm{~mm}$ slice thickness, 192 imaging acquisition matrix, repetition and echo time 
A

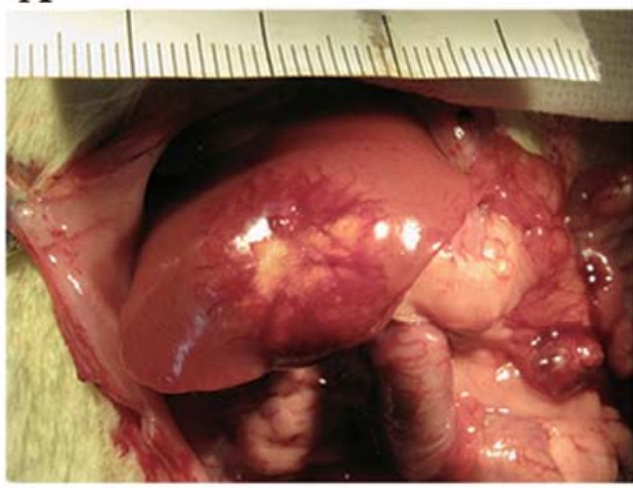

B

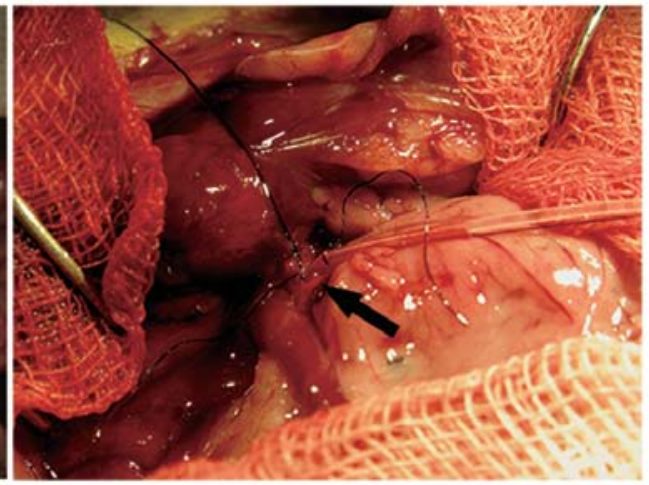

Figure 1. Catheterization and TAE performed on a rat. (A) TAE was performed two weeks after implantation when the liver tumor reached $\sim 1 \mathrm{~cm}$ in diameter. (B) A silastic catheter was inserted in a retrograde manner into the gastroduodenal artery (black arrow) and extended into the common hepatic artery.

$(\mathrm{TR} / \mathrm{TE})=3500 / 103 \mathrm{~ms}$ and total examination time, $4 \mathrm{~min}$ $5 \mathrm{sec}$. The volume of the tumors was calculated as follows: $\left(\mathrm{L} \times \mathrm{S}^{2}\right) / 2$, where $\mathrm{L}$ is the longest diameter and $\mathrm{S}$ is the shortest diameter. All animals were euthanized 2 weeks after TAE, and their livers and lungs were harvested to evaluate molecular changes and distant metastasis. Lung metastases were visualized using fluorescence stereomicroscopy (Leica Microsystems Imaging Solutions, Cambridge, UK). Part of tumor tissues were frozen in liquid nitrogen for western blot analysis, while other parts were fixed in $10 \%$ formalin, embedded in paraffin and sectioned $(3 \mu \mathrm{m})$ for histopathological studies.

Western blot assays. The concentration of proteins extracted from cell specimens (treatment with or without $\mathrm{CoCl}_{2}$ ) and tumor tissues was determined using the BCA Protein Assay kit (Beyotime Institute of Biotechnology, Shanghai, China). Then, the expression of HIF-1 $\alpha$, E-cadherin, N-cadherin and vimentin (monoclonal antibodies; Bioworld Technology) was determined by western blotting according to the manufacturer's instructions. The density of each band was measured and compared to that of the internal control, GAPDH.

Immunohistochemistry and hematoxylin and eosin $(H \& E)$ staining. Tissue sections were used for $\mathrm{H} \& \mathrm{E}$ staining and immunostaining of HIF-1 $\alpha$, E-cadherin, N-cadherin and vimentin. Three fields (x400 magnification) from each slide were counted to determine the frequency of nuclear or cytoplasmic staining. The expression of each target protein was assessed according to the percentage of immunoreactive cells within the region of neoplastic cells. All slides were evaluated by 2 pathologists in a blinded manner under a light microscope and divergent results were resolved by discussion.

Statistical analysis. The cell invasion and proliferation assays were analyzed using the Student's t-test. Tumor volume, lung metastasis, western blot assays and immunohistochemistry staining were analyzed using t-tests or Wilcoxon rank-sum tests. Statistical analysis was performed with SPSS (Statistical Package for the Social Sciences) 15.0 for Windows (SPSS Inc., Chicago, IL, USA). P $<0.05$ was considered to indicate a statistically significant result.

\section{Results}

Hypoxia promotes the invasiveness and increases the expression of EMT markers in McA-RH7777 cells. The analysis of tumor cell proliferation at 24 and $48 \mathrm{~h}$ after hypoxia induced by $\mathrm{CoCl}_{2}$ showed that hypoxia inhibited cell proliferation in comparison to the normoxic controls. In addition, the morphology of hypoxic cells was altered from a typical epithelial orbicular-ovate appearance to a tenuous/irregular shape with formation of pseudopodia, demonstrating reduced cell-cell adhesion (Fig. 2A). The results of the Transwell assay showed that the number of hypoxic tumor cells that invaded through the Matrigel was significantly higher than the corresponding number of normoxic cells (30.2 \pm 2.46 vs. 20.4 \pm 1.89 ; $\mathrm{P}=0.013$ ) (Fig. 2B).

Immunoblotting demonstrated that hypoxia was successfully induced with $\mathrm{CoCl}_{2}$, as indicated by the increase in HIF-1 $\alpha$ expression, which was significantly higher in the hypoxic as compared to the normoxic group (Fig. 3A and B). Accompanying morphological changes, the western blot analysis demonstrated reduced expression of the epithelial cell marker E-cadherin in hypoxic cells relative to normoxic cells. Furthermore, the level of the mesenchymal cell markers vimentin and $\mathrm{N}$-cadherin was increased (Fig. 3A and B). This phenomenon was related to EMT, which is characterized by the loss of E-cadherin expression and the simultaneous upregulation of $\mathrm{N}$-cadherin, which results in the ability of cells to transmigrate from basement membranes into stromal tissues.

The growth characteristics and imaging properties of the hepatoma model. Tumor implantation led to the outgrowth of solitary liver tumors in all 20 animals. None of the animals died during implantation or during the postoperative period. Seven days after orthotopic liver tumor implantation, the orthotopic liver tumors were so small that it was difficult to assess the tumors using MRI in most cases. Two weeks after implantation, the liver tumors reached $\sim 1 \mathrm{~cm}$ in diameter $(8.15 \pm 0.13 \mathrm{~mm})$ and showed high signals on $\mathrm{T}_{2} \mathrm{WI}$ imaging (Fig. 4A). The tumors were sharply demarcated from the surrounding normal hepatic parenchyma with an incomplete capsule. On $\mathrm{T}_{2} \mathrm{WI}$ imaging, the necrotic tissue was observed 

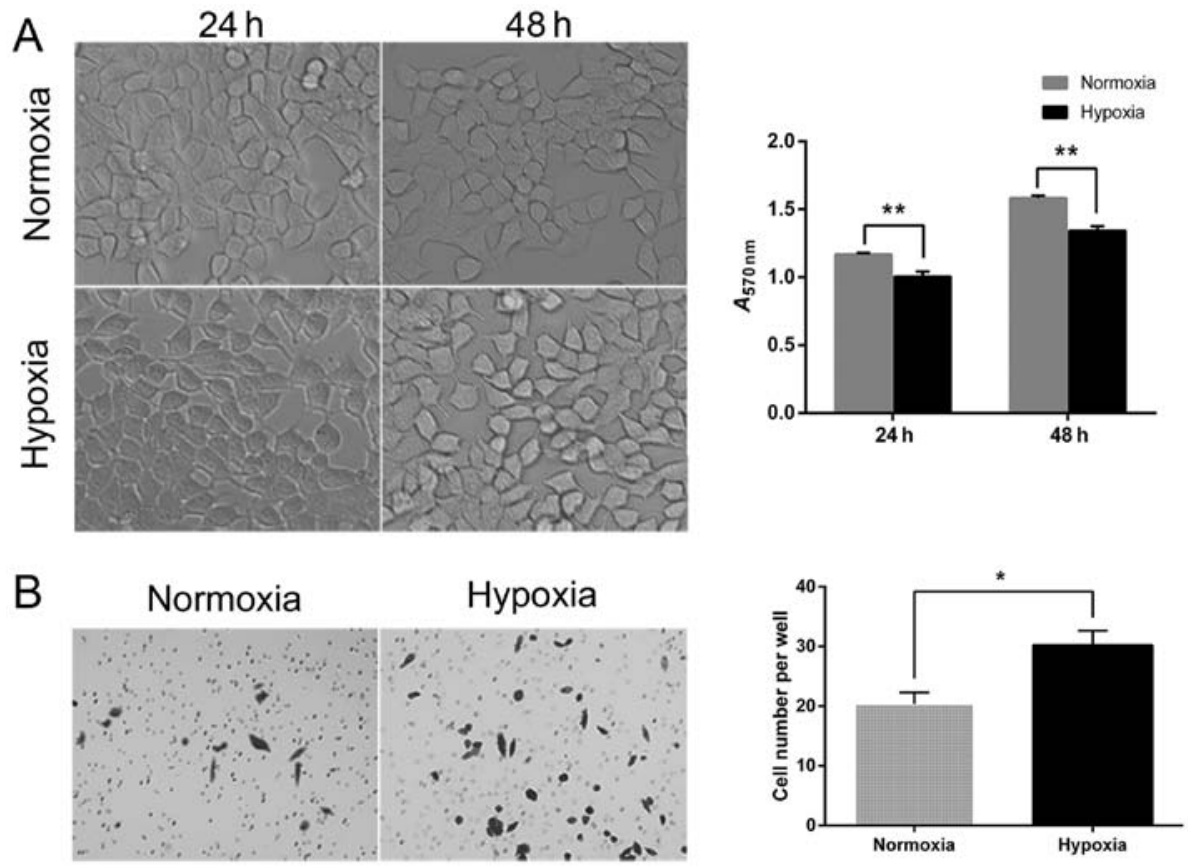

Figure 2. Hypoxia promotes cell invasion but inhibits cell proliferation (magnification, $\mathrm{x} 200$ ). (A) At 24 and $48 \mathrm{~h}$ after hypoxia induced by CoCl${ }_{2}$, cells had an altered appearance, changing from an orbicular-ovate shape at normoxia to either a spindle or irregular shape at hypoxia. The CCK- 8 analysis showed that the $\mathrm{A}_{570 \mathrm{~nm}}$ of the hypoxic group was lower than that of the control, respectively. (B) The invasion of cancer cells was measured by Transwell assays, and significant enhancement of cell invasion was found in hypoxic tumor cells, compared with normoxic cells.
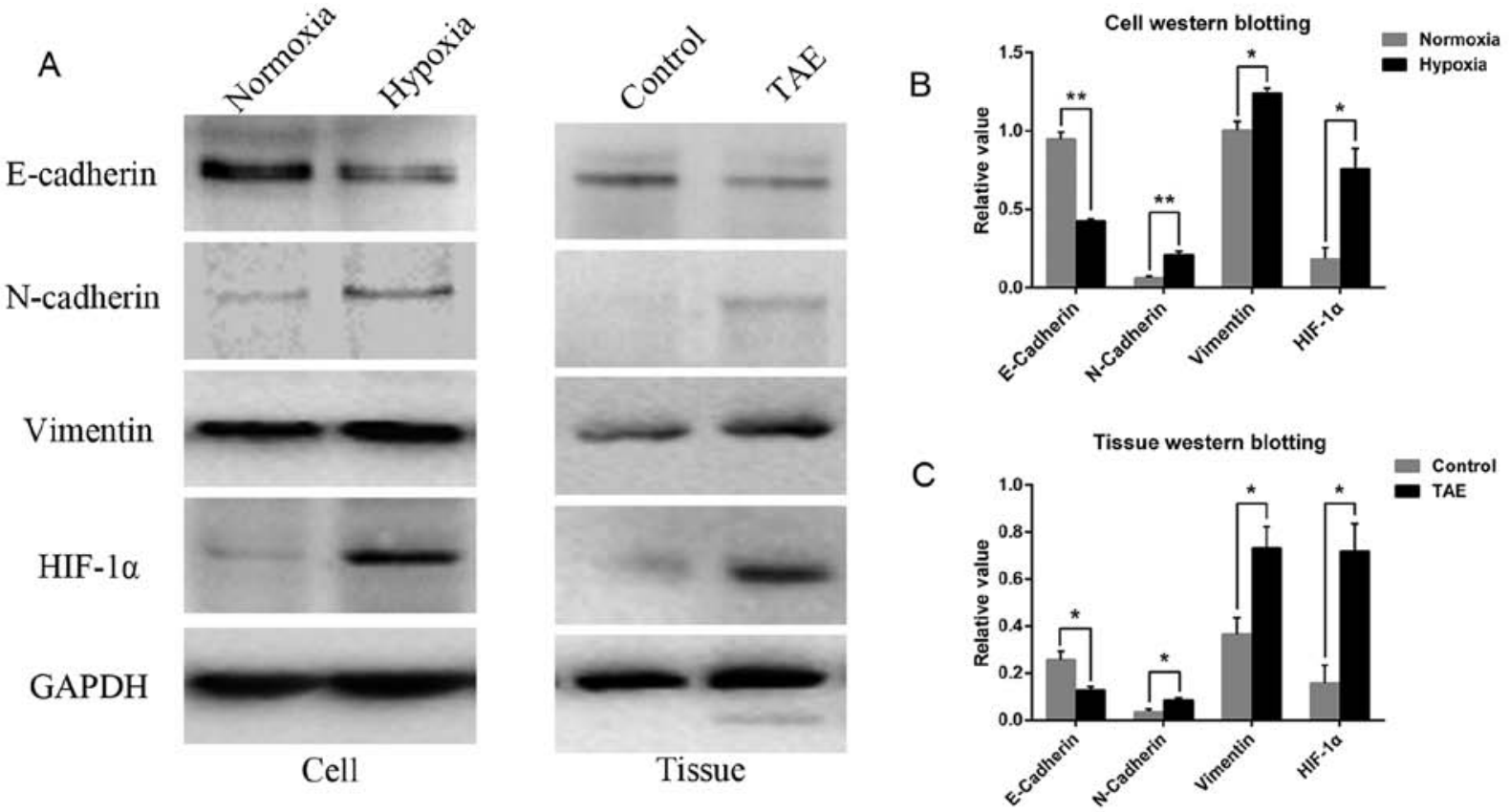

Figure 3. Hypoxia induces EMT in vitro and in vivo. (A) Western blot analysis showed that molecular changes relating to EMT (downregulation of E-cadherin and upregulation of N-cadherin and vimentin) were induced by hypoxia in (B) cell and (C) after TAE in tissue, which were accompanied by an increase in HIF-1 $\alpha$ expression. GAPDH was used as an internal control.

as an area of lower intensity in the center. No significant difference was found in pretreatment tumor size between the TAE-treated and control groups $\left(208.56 \pm 13.62 \mathrm{~mm}^{3} \mathrm{vs}\right.$. $193.88 \pm 12.32 \mathrm{~mm}^{3} ; \mathrm{P}=0.435$ ).

TAE inhibits tumor growth but significantly promotes metastases. TAE was performed successfully in all 20 animals, and no technical complications occurred during the experimental period. Two weeks after TAE, the tumor volume of the TAE group was $2,052.20 \pm 178.37 \mathrm{~mm}^{3}$, which was smaller than that of sham-operated controls $\left(3,339.50 \pm 306.18 \mathrm{~mm}^{3} ; \mathrm{P}=0.002\right)$ (Fig. 4). However, the lung metastatic rate of the TAE group was the same as the sham-operated group $(100 \%, 6 / 6)$. Additionally, the TAE group showed more lung metastatic 
A

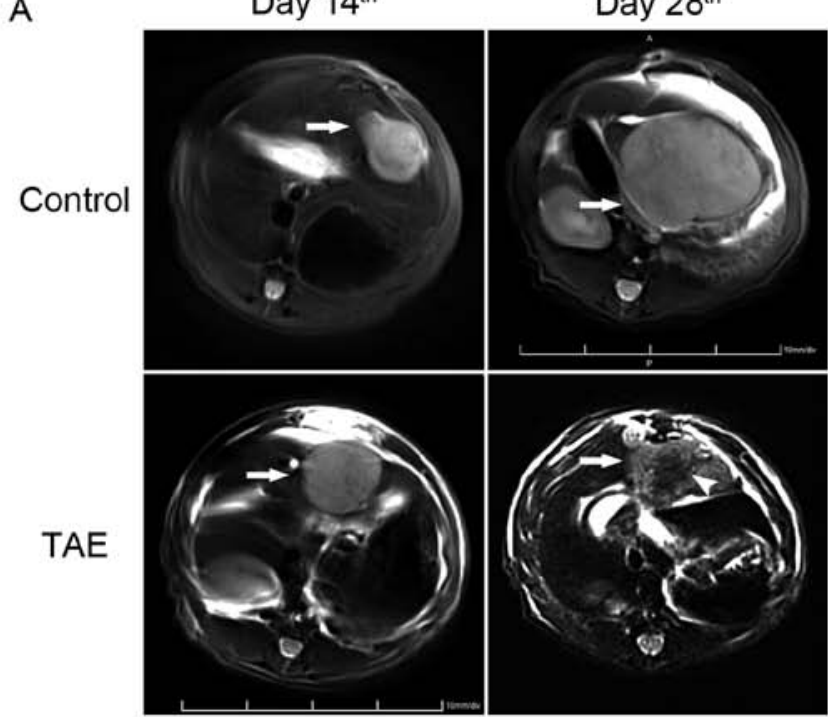

B

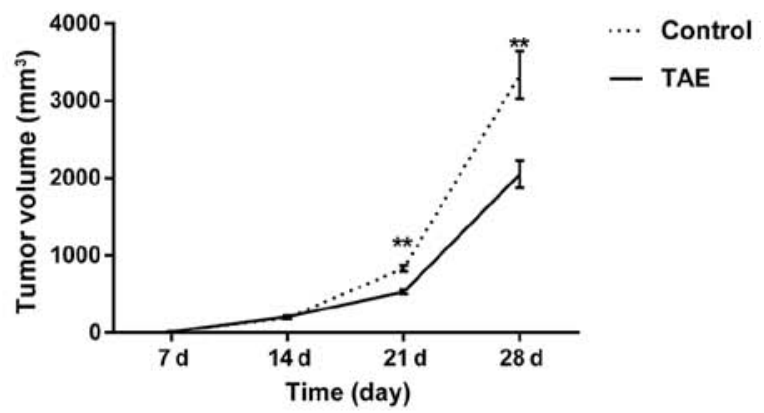

Figure 4. The growth of rat orthotopic hepatoma investigated by MRI. (A) On $\mathrm{T}_{2} \mathrm{WI}$, the liver tumors (white arrow) which showed high signal were sharply demarcated from the surrounding normal hepatic parenchyma. Two weeks after TAE, the growth of tumor in TAE group was inhibited. The tumor was shrunken and necrosis was observed in the center tumor (lower intensity, arrow head) after TAE. (B) The time-volume curve for the tumors showed that the increase in tumor volume was less than that observed in the control after TAE.

A
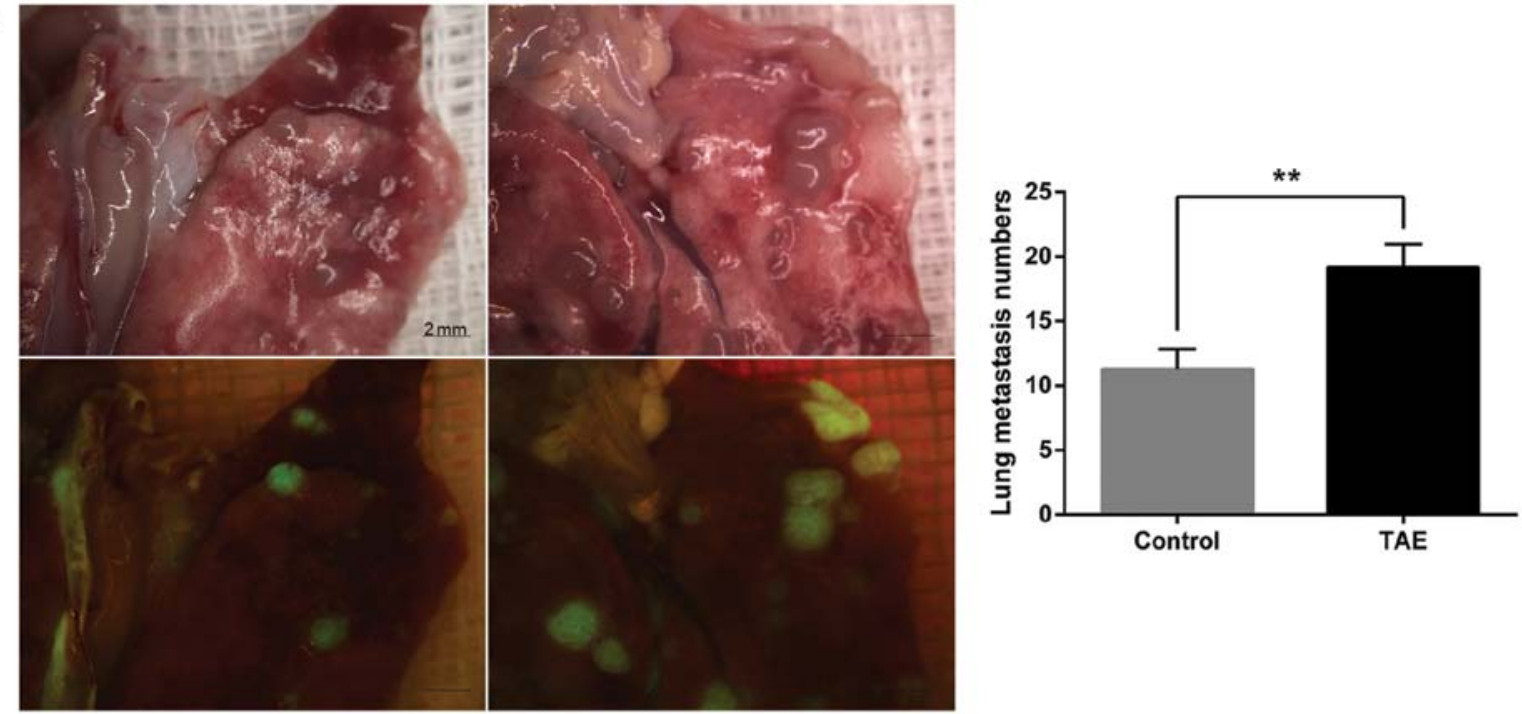

B

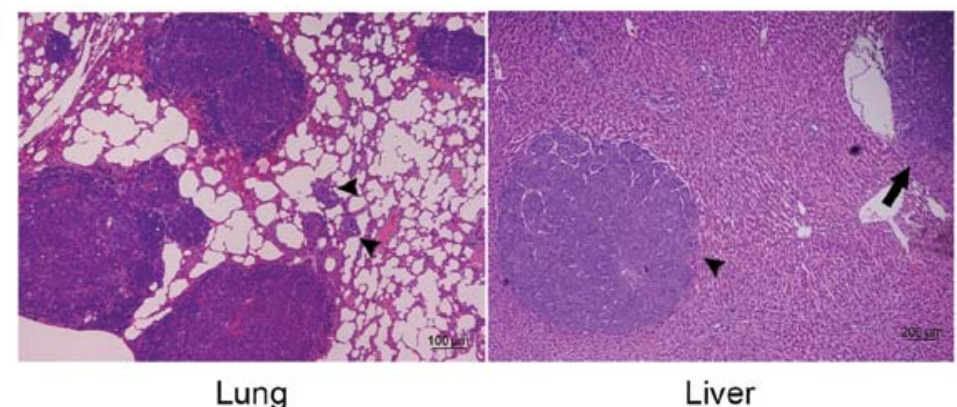

Figure 5. TAE promotes lung metastases and intrahepatic dissemination. (A) GFP fluorescence showed that the number of lung nodules in the TAE group was significantly greater than that in the control group $(\mathrm{P}=0.003)$. (B) The microscopic lung metastases could be observed in rat belongs to TAE group (black arrow head). The microscopic intrahepatic dissemination was also present in the TAE group (black arrow head), adjacent to the primary tumor (black arrow),

nodules in comparison to the sham-operated group $(19.20 \pm 1.76$ vs. $11.30 \pm 1.54 ; \mathrm{P}=0.003$ ) by GFP fluorescence (Fig. $5 \mathrm{~A}$ ). Furthermore, the proinvasive consequences of TAE could be observed in animals bearing McA-RH7777 homografts, and microscopic intrahepatic dissemination was also present in the TAE group (Fig. 5B). 

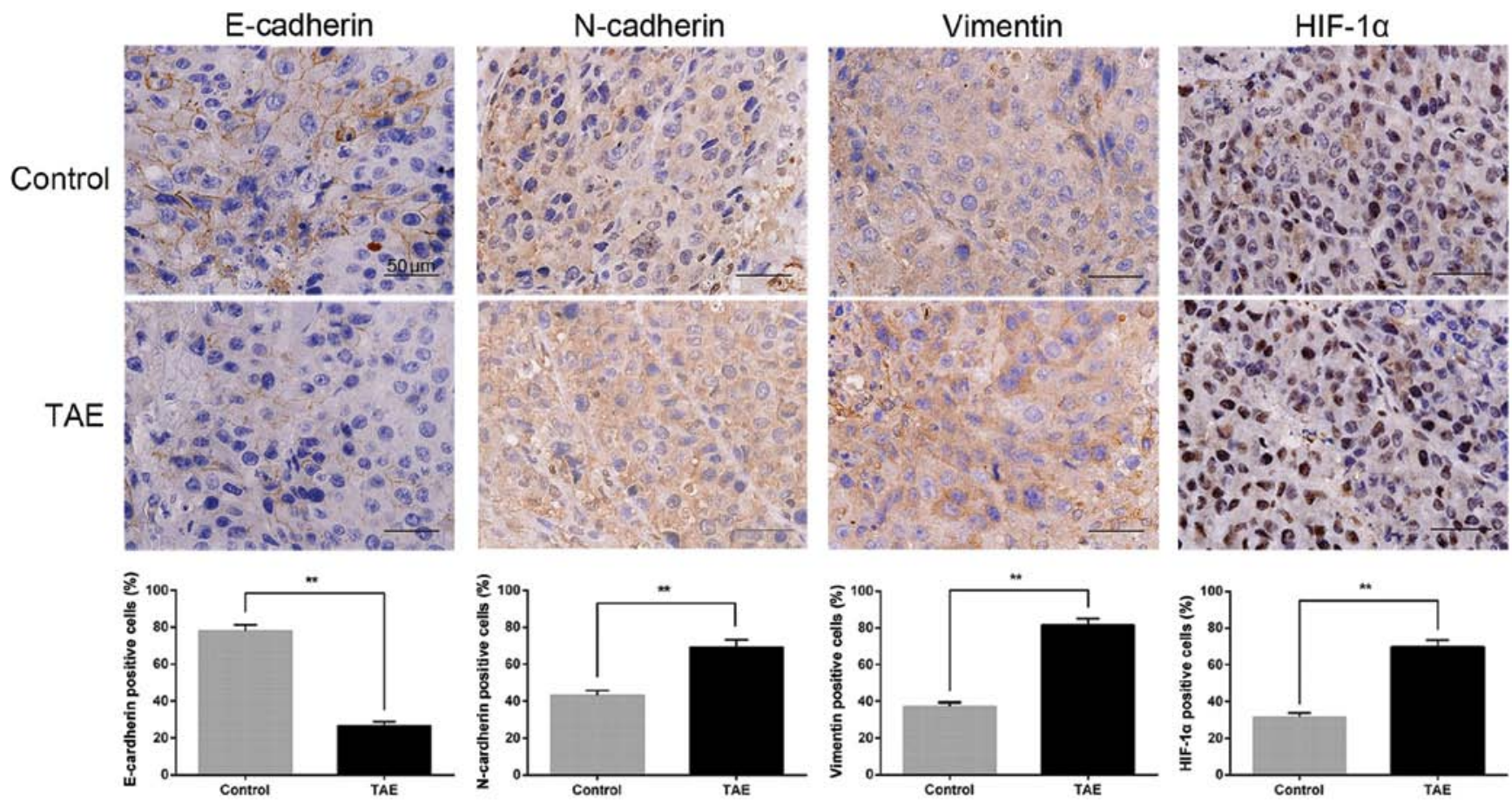

Figure 6. EMT induces by hypoxia following TAE. Immunostaining (magnification, $x 400$ ) showed that downregulation of E-cadherin and upregulation of $\mathrm{N}$-cadherin and vimentin were induced accompanying the increase in HIF-1 $\alpha$ expression that occurred after TAE.

TAE induces changes consistent with EMT in HCC. After TAE, the increase in HIF-1 $\alpha$ expression and induction of EMT were demonstrated by immunoblotting, characterized by the loss of E-cadherin and upregulation of $\mathrm{N}$-cadherin and vimentin in the tumor tissues of TAE-treated rats (Fig. 3A and $\mathrm{C}$ ). Immunostaining showed that E-cadherin-positive cells in sham-operated mice were localized in the tumor areas and showed a membranous distribution. Furthermore, this analysis also revealed a trend towards enhanced expression of HIF-1 $\alpha$, which showed a nuclear distribution in TAE-treated rats in comparison to the corresponding sham-operated controls, as well as increased expression of $\mathrm{N}$-cadherin and vimentin (Fig. 6).

\section{Discussion}

The aim of TAE for liver tumors is the induction of ischemic/hypoxic tumor necrosis through embolization of the tumor-feeding vessel. Considering patients' reserved liver function and the complexity of the blood supply for liver tumors, embolization of the tumor-feeding vessel is always incomplete and tumor necrosis can be partial, which allows residual tumor to survive and adapt to a microenvironment of hypoxia. Moreover, the merits of TACE or TAE for HCC are disputable because of the possibility of increasing the likelihood of metastasis, and some findings have ascribed the failure of TAE to embolization-induced hypoxia (27). In the present study, we used McA-RH7777 hepatoma-bearing rats to mimic incomplete embolization and investigate the influence of hypoxia after TAE on the invasiveness and metastatic potential of residual carcinoma.

Hypoxia presents fundamental implications in many cancers. In fact, part of the tumor is almost always hypoxic, which increases as tumors grow rapidly, especially in the tumor core due to insufficient vascularization. The consequences of hypoxia for tumor cells vary according to the extent and duration of hypoxia. Severe hypoxia causes cell death, whereas on the other hand, mild hypoxia can induce a series of adaptive changes, such as angiogenesis (28). The transcription factor HIF-1, an oxygen-sensitive transcriptional activator, plays an important role in antagonizing apoptosis and mediating adaptive cell responses to hypoxia following TAE. HIF-1 is a heterodimer that consists of an oxygen-regulated HIF- $1 \alpha$ subunit and a constitutively expressed HIF-1 $\beta$ subunit. In normoxia, the HIF-1 $\alpha$ proteins are rapidly degraded, whereas in hypoxia, HIF-1 $\alpha$ becomes stabilized and free to form dimers with HIF-1 $\beta$, and the HIF-1 complex then translocates to the nucleus, where it regulates the transcription of genes involved many key biological functions (29). Moreover, studies on tumor angiogenesis, cell survival/apoptosis, invasion, metastasis and adhesion have revealed a critical role for HIF- $1 \alpha$ in these processes $(30,31)$. Because of the limitations of TACE in comparison to radical resection, a protective response against ischemic/hypoxic injury arises in the residual viable tumor tissue. Significant evidence indicates that HIF-1 $\alpha$ serves as a prognostic factor for tumor recurrence in human and murine HCC and that high expression of HIF-1 $\alpha$ at the edge of a tumor may correlate with migration and invasion (32). Consistent with previous studies, we showed that HIF-1 $\alpha$ was overexpressed as a result of hypoxia generated by the TAE procedure in vivo. Furthermore, we investigated the effect of hypoxia induced by $\mathrm{CoCl}_{2}$ in vitro, and the increase in HIF-1 $\alpha$ protein demonstrated the successful induction of a hypoxic microenvironment, analogous to that resulting from TAE. 
Our findings further demonstrated that hypoxia, mediated by HIF-1 $\alpha$, led to increased motility and invasiveness of residual viable tumor tissue. The acquisition of motile and invasive abilities represents a critical step in metastasis, and the escape of carcinoma cells from the primary tumor generally occurs along with the loss of cell-to-cell contacts and the degradation of the extracellular matrix (ECM), a process referred to as EMT. In fact, EMT is the key process that drives cancer metastasis and is characterized by loss of the epithelial marker E-cadherin; increased expression of the mesenchymal markers vimentin, $\mathrm{N}$-cadherin and ICAM-1; and enhanced metastatic potential of tumor cells $(22,33)$. Our results showed that hypoxia stimulated the transformation of HCC cells from a typical epithelial phenotype to a spindle-shaped mesenchymal phenotype, accompanied by the loss of E-cadherin and upregulation of $\mathrm{N}$-cadherin and vimentin. These observations imply that HIF-1 $\alpha$ may play a role in EMT. After the initiation of hypoxia, tumor cells that have undergone EMT are endowed with the ability to transmigrate across basement membranes and stromal tissues as well as to intravasate and pass into the circulatory system. These changes increase the likelihood of recurrence and metastasis, and accordingly, the number of lung metastases in the TAE group was significantly higher than that observed in the sham group.

In contrast to other studies that have focused on the therapeutic effects of TAE on primary tumor growth, with less attention on metastasis, we illustrated the significance of incomplete TAE on tumor growth and invasiveness in an orthotopic tumor implantation rat model. Although the growth of allografts was inhibited after TAE, the residual tumors were more invasive, and this result supports the relationship between intratumoral hypoxia and tumor cell EMT. Following the introduction of incomplete TAE, our results indicated that the non-selective embolization of blood flow could elicit an adaptive response in hepatic tumor cells, involving an augmented invasive ability, malignant phenotype, increased intrahepatic dissemination and even distant metastasis.

In general, metastasis, rather than growth of the primary tumor, represents the major cause of cancer-related mortality (34). Although TACE has been established as the standard of care for patients who meet the criteria for the intermediate stage of the BCLC staging system (1), high metastasis and recurrence rates following TACE as well as other treatments drive physicians to exploit combined strategies. Given that VEGF expression and VEGF-receptor activation are markedly increased in HCC after TACE $(35,36)$, and these observations are widely accepted as indicative of tumor degradation and metastasis, anti-angiogenic or anti-VEGF agents have been administered in combination with embolization (37). Recent investigations have also demonstrated encouraging results in terms of survival following combined therapy consisting of TACE and sorafenib (38). However, angiogenesis may, at least in part, account for the failure of embolization. Alternatively, hypoxia may induce EMT, and this process is known to convert adherent epithelial cells into migratory cells that invade the ECM and has consistently been associated with tumor metastasis $(33,39)$. Notably, embolization of the hepatic artery using lipiodol induced EMT in hepatic tumor cells in the present study, and this process was linked to the hypoxic response. Thus, our results seem to be comparable to those of previous in vivo studies of EMT in hypoxic ovarian, breast, renal and hepatic tumor cells. However, we also demonstrated that EMT contributed to the depravation of HCC after TAE, which has only rarely been reported, and these findings may provide important evidence concerning the clinical application of TACE for HCC patients.

There were several limitations of the present study. First, because tumor metastasis is influenced by numerous molecular mechanisms and microcirculation factors and because the sample sizes were limited in the present study, the contribution of hypoxia to EMT warrants further investigation and should be studied in combination with chemotherapeutic agents. Second, we did not assess the correlation between HIF-1 $\alpha$ protein and EMT at different time points after TAE, although it has been shown that HIF-1 $\alpha$ activity changes dynamically and that VEGF expression and the microvessel density are significantly increased in liver tumors 2-3 days after TAE (35). Thus, the degree of EMT may vary at different time-points, and further study with optimized time points of analysis is warranted. Third, experimental rats such as those used in the present study can develop severe liver dysfunction, and because the effect of embolization was limited using nonselective catheterization, we did not evaluate the degree of tumor necrosis after TAE.

In conclusion, we have shown that HIF-1 $\alpha$ protein is increased in liver tumors after TAE as a result of the local hypoxia induced by the procedure and that this process is involved in the activation of EMT in the residual viable tumor. Similarly, hypoxia after TACE may increase the invasiveness and metastatic potential of residual HCC tumors in clinical settings, and combination therapy with targeted molecular agents against EMT induced by hypoxia may augment the therapeutic effects of TACE.

\section{Acknowledgements}

We thank Zuxing Kan (MD Anderson Cancer Center, Houston, TX, USA) for technical support and executive assistance. The present study was supported in part by the grants from the National Natural Sciences Foundation of China (no. 81171432).

\section{References}

1. EASL-EORTC clinical practice guidelines: management of hepatocellular carcinoma. J Hepatol 56: 908-943, 2012.

2. El-Serag HB: Hepatocellular carcinoma. N Engl J Med 365: 1118-1127, 2011.

3. Forner A, Llovet JM and Bruix J: Hepatocellular carcinoma. Lancet 379: 1245-1255, 2012.

4. Hasegawa K, Makuuchi M, Takayama T, et al: Surgical resection vs. percutaneous ablation for hepatocellular carcinoma: a preliminary report of the Japanese nationwide survey. J Hepatol 49: 589-594, 2008

5. Chen MS, Li JQ, Zheng Y, et al: A prospective randomized trial comparing percutaneous local ablative therapy and partial hepatectomy for small hepatocellular carcinoma. Ann Surg 243: 321-328, 2006

6. Llovet JM, Ricci S, Mazzaferro V, et al: Sorafenib in advanced hepatocellular carcinoma. N Engl J Med 359: 378-390, 2008.

7. Fernandez M, Semela D, Bruix J, Colle I, Pinzani M and Bosch J: Angiogenesis in liver disease. J Hepatol 50: 604-620, 2009.

8. Llovet JM, Schwartz M and Mazzaferro V: Resection and liver transplantation for hepatocellular carcinoma. Semin Liver Dis 25: $181-200,2005$. 
9. Sala M, Fuster J, Llovet JM, et al: High pathological risk of recurrence after surgical resection for hepatocellular carcinoma: an indication for salvage liver transplantation. Liver Transpl 10: 1294-1300, 2004.

10. Llovet JM and Bruix J: Systematic review of randomized trials for unresectable hepatocellular carcinoma: chemoembolization improves survival. Hepatology 37: 429-442, 2003.

11. Lo CM, Ngan H, Tso WK, et al: Randomized controlled trial of transarterial lipiodol chemoembolization for unresectable hepatocellular carcinoma. Hepatology 35: 1164-1171, 2002.

12. Virmani S, Rhee TK, Ryu RK, et al: Comparison of hypoxiainducible factor- $1 \alpha$ expression before and after transcatheter arterial embolization in rabbit VX2 liver tumors. J Vasc Interv Radiol 19: 1483-1489, 2008.

13. Wang $\mathrm{B}, \mathrm{Xu} \mathrm{H}$, Gao ZQ, Ning HF, Sun YQ and Cao GW: Increased expression of vascular endothelial growth factor in hepatocellular carcinoma after transcatheter arterial chemoembolization. Acta Radiol 49: 523-529, 2008.

14. Sergio A, Cristofori C, Cardin R, et al: Transcatheter arterial chemoembolization (TACE) in hepatocellular carcinoma (HCC): the role of angiogenesis and invasiveness. Am J Gastroenterol 103: 914-921, 2008.

15. Shim JH, Park JW, Kim JH, et al: Association between increment of serum VEGF level and prognosis after transcatheter arterial chemoembolization in hepatocellular carcinoma patients. Cancer Sci 99: 2037-2044, 2008 .

16. Friedl $\mathrm{P}$ and Wolf $\mathrm{K}$ : Tumour-cell invasion and migration: diversity and escape mechanisms. Nat Rev Cancer 3: 362-374, 2003.

17. Thiery JP and Sleeman JP: Complex networks orchestrate epithelial-mesenchymal transitions. Nat Rev Mol Cell Biol 7: 131-142, 2006.

18. Polyak K and Weinberg RA: Transitions between epithelial and mesenchymal states: acquisition of malignant and stem cell traits. Nat Rev Cancer 9: 265-273, 2009.

19. van Zijl F, Zulehner G, Petz M, et al: Epithelial-mesenchymal transition in hepatocellular carcinoma. Future Oncol 5: $1169-1179,2009$

20. Choi SS and Diehl AM: Epithelial-to-mesenchymal transitions in the liver. Hepatology 50: 2007-2013, 2009.

21. Copple BL: Hypoxia stimulates hepatocyte epithelial to mesenchymal transition by hypoxia-inducible factor and transforming growth factor- $\beta$-dependent mechanisms. Liver Int 30: 669-682, 2010.

22. Thiery JP: Epithelial-mesenchymal transitions in tumour progression. Nat Rev Cancer 2: 442-454, 2002.

23. De Larco JE, Wuertz BR, Manivel JC and Furcht LT: Progression and enhancement of metastatic potential after exposure of tumor cells to chemotherapeutic agents. Cancer Res 61: 2857-2861, 2001
24. Ankoma-Sey V, Wang Y and Dai Z: Hypoxic stimulation of vascular endothelial growth factor expression in activated rat hepatic stellate cells. Hepatology 31: 141-148, 2000.

25. Kan Z, Phongkitkarun S, Kobayashi S, et al: Functional CT for quantifying tumor perfusion in antiangiogenic therapy in a rat model. Radiology 237: 151-158, 2005.

26. Maataoui A, Qian J, Mack MG, et al: Liver metastases in rats: chemoembolization combined with interstitial laser ablation for treatment. Radiology 237: 479-484, 2005.

27. Ramsey DE, Kernagis LY, Soulen MC and Geschwind JF: Chemoembolization of hepatocellular carcinoma. J Vasc Interv Radiol 13: S211-S221, 2002.

28. Harris AL: Hypoxia - a key regulatory factor in tumour growth. Nat Rev Cancer 2: 38-47, 2002.

29. Ivan M, Kondo K, Yang H, et al: HIF $\alpha$ targeted for VHL-mediated destruction by proline hydroxylation: implications for $\mathrm{O}_{2}$ sensing. Science 292: 464-468, 2001 .

30. Brahimi-Horn MC, Chiche J and Pouyssegur J: Hypoxia and cancer. J Mol Med (Berl) 85: 1301-1307, 2007.

31. Sendoel A, Kohler I, Fellmann C, Lowe SW and Hengartner MO: HIF-1 antagonizes p53-mediated apoptosis through a secreted neuronal tyrosinase. Nature 465: 577-583, 2010.

32. Daskalow K, Rohwer N, Raskopf E, et al: Role of hypoxia-inducible transcription factor $1 \alpha$ for progression and chemosensitivity of murine hepatocellular carcinoma. J Mol Med (Berl) 88: 817-827, 2010.

33. Thiery JP, Acloque H, Huang RY and Nieto MA: Epithelialmesenchymal transitions in development and disease. Cell 139: 871-890, 2009

34. Gupta GP and Massague J: Cancer metastasis: building a framework. Cell 127: 679-695, 2006.

35. Gupta S, Kobayashi S, Phongkitkarun S, Broemeling LD and Kan Z: Effect of transcatheter hepatic arterial embolization on angiogenesis in an animal model. Invest Radiol 41: 516-521, 2006.

36. Liang B, Zheng CS, Feng GS, et al: Correlation of hypoxiainducible factor $1 \alpha$ with angiogenesis in liver tumors after transcatheter arterial embolization in an animal model. Cardiovasc Intervent Radiol 33: 806-812, 2010.

37. Maataoui A, Qian J, Vossoughi D, et al: Transarterial chemoembolization alone and in combination with other therapies: a comparative study in an animal HCC model. Eur Radiol 15: $127-133,2005$.

38. Abou-Alfa GK: TACE and sorafenib: a good marriage? J Clin Oncol 29: 3949-3952, 2011.

39. Acloque H, Adams MS, Fishwick K, Bronner-Fraser $M$ and Nieto MA: Epithelial-mesenchymal transitions: the importance of changing cell state in development and disease. J Clin Invest 119: 1438-1449, 2009. 